\title{
Characterization of Optimum Polarization for Multiple Target Discrimination Using Genetic Algorithms
}

\author{
Kamal Sarabandi, Senior Member, IEEE, and Eric S. Li
}

\begin{abstract}
In this paper, a stochastic optimization algorithm is used to characterize the polarization states of a nonpolarimetric radar transmitter and receiver antennas for optimal target classification. Specifically, the optimized solution is sought when a multitude of targets are to be categorized. It is shown that the objective function of the optimization problem is highly nonlinear and discontinuous, hence, classical optimization algorithms fail to provide satisfactory results. The stochastic optimization algorithm used in this paper is based on a genetic algorithm (GA) which operates on a discretized form of the parameter space and searches globally for the optimum point. In this process, it is assumed that the polarimetric responses of the targets are known a priori. The optimization algorithm is applied to two sets of data: 1) a synthetic backscatter data for four point targets with similar radar cross sections (RCS's) and 2) a set of polarimetric backscatter measurements of asphalt surfaces under different physical conditions at $94 \mathrm{GHz}$. The purpose of the latter study is to come up with the optimal design for polarization states of an affordable millimeter-wave radar sensor that can assess traction of road surfaces.
\end{abstract}

Index Terms-Genetic algorithms, object detection.

\section{INTRODUCTION}

$\mathbf{I}$ $\mathrm{N}$ radar scattering problems in which target discrimination cannot be accomplished on the basis of a difference in range, the polarization spectrum (signature) may be the appropriate tool to differentiate between different targets or targets and clutter. This idea stems from the fact that the polarization for which the radar receives the maximum or minimum scattered power (optimum polarization) from a target may be different from the optimum polarizations of the other targets or its surrounding clutter. The literature concerning application of wave polarization for enhancing target detection dates back to about five decades ago with the pioneering work of Kennaugh and Huynen [1], [2]. The concept of optimal polarization has long been used by radar engineers to reduce the effect of clutter such as hydrometeors in reconnaissance radars [3], [4]. Since the early work of Kennaugh, much effort has been devoted to utilize the polarimetric responses of targets in inverse problems [5] and in a variety of remote sensing problems [6], [7]. Also, procedures for evaluating the statistics

Manuscript received July 25, 1996; revised August 6, 1997. This work was supported by the National Automotive Highway System Consortium (NAHSC) and the U.S. Army Tank Automotive Command.

The authors are with the Radiation Laboratory, Department of Electrical Engineering and Computer Science, The University of Michigan, Ann Arbor, MI 48109 USA.

Publisher Item Identifier S 0018-926X(97)09047-9. of polarimetric backscatter responses of distributed targets and their relationship with the target physical parameters have been investigated intensively [8]-[11].

In this paper, we confine our interest to the problem of characterizing polarization combination of the receive and transmit antennas of a radar system for target classification. Reported polarimetric techniques for target discrimination have been limited to the special case of two targets because of the complex nature of the optimization problem. For example, in the method proposed by Ioannidis and Hammers [12] the optimum polarizations are obtained from a constrained maximization of two bilinear forms representing the signalto-clutter backscatter ratio. In another method suggested by van Zyl et al. [13], a comparison based on the normalized root mean square (rms) of difference in the received powers for two targets has been suggested. In this method, the received powers are calculated for $N$ polarization configurations ( $N$ points on the Poincare sphere) to obtain the rms of power difference. The motivation for considering the problem at hand stems from the fact that in many cases of practical importance, target classification, when there are more than two possible targets, is of interest. The complex optimization problem in finding the receive and transmit polarizations of a nonpolarimetric radar will be addressed using a stochastic optimization method.

Stochastic algorithms such as simulated annealing [14] and genetic algorithms (GA's) [15], [16] offer an alternative for the traditional gradient-based optimization methods where the dimension of parameter space is large and/or the objective function is nondifferentiable. In recent years, applications of GA's to a variety of optimization problems in electromagnetics have been successfully demonstrated [17]-[19]. The fundamental concept of GA's is based on natural selection in the evolution process, which is accomplished by genetic recombination and mutation. In this approach, the entire parameter space is discretized and using a Monte Carlo simulation of the evolution process on a randomly selected subset of the discretized parameter space, the desired objective function is optimized. GA's offer certain advantages over the traditional gradient-based (TGB) optimization algorithms. The most important feature of GA's is that the optimization is accomplished globally; that is, the probability of converging to a weak local minimum is very low unlike the TGB algorithms. This is particularly the case when the objective function is highly nonlinear and the dimension of the parameter space is large. GA's perform equally well independent of the objective 


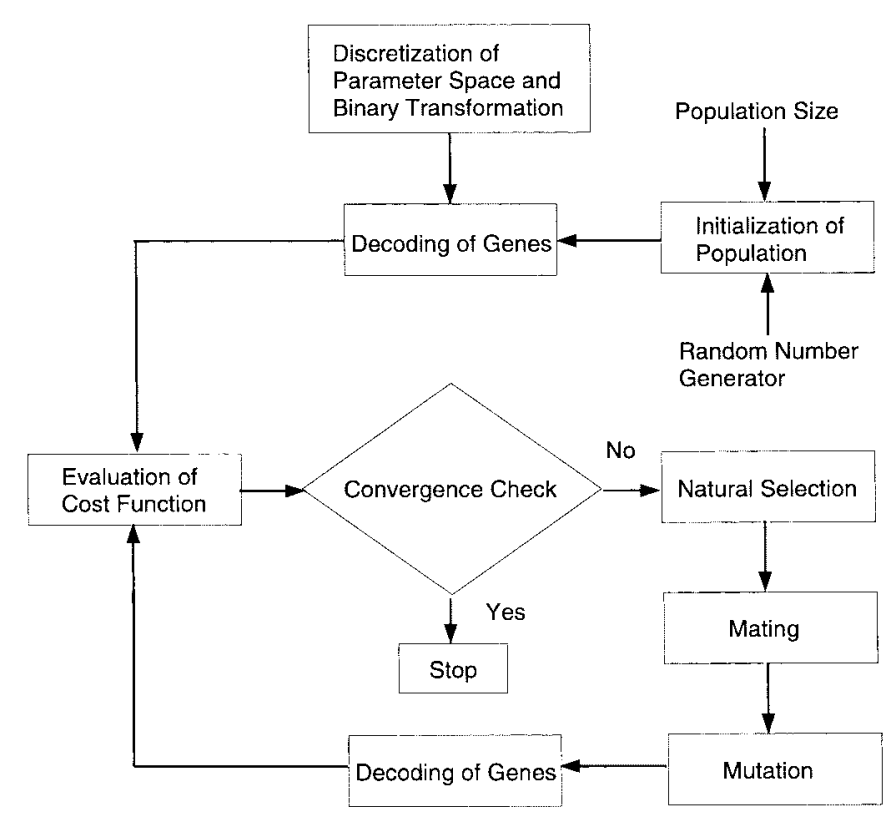

Fig. 1. Flow chart of the GA.

function's smoothness condition and after convergence provide a list of high-quality solutions, which can further be assessed according to criteria not included in the objective function. On the other hand, there are certain disadvantages associated with the GA's. A major drawback of the GA's is their lack of computational efficiency. Basically, far more calculation of the objective function is required to achieve a convergence when compared with TGB's. Another shortcoming of the GA's is that they do not provide any insight as to the character of the objective function during the course of the optimization process. It should also be noted that after convergence the solution may not necessarily be the true extremum of the objective function.

In this paper, application of GA's for characterization of optimum antenna polarizations of a nonpolarimetric radar is considered to achieve multiple target classification with least-error probability. The objective function is defined so that the solution will directly specify the threshold levels for target classification. In this procedure, it is assumed that the polarimetric responses of the targets are known. The paper is organized as follows. First the objective function of the optimization problem is described. In Section III, the procedure for implementing a GA for the problem at hand is provided. In Section IV, performance of GA's in characterizing the optimum polarizations is presented. In this section, both point targets and distributed targets are considered.

\section{Problem Formulation}

As mentioned earlier, the objective of the optimization problem is characterization of the optimum polarizations of a nonpolarimetric radar for target classification. Apart from the classical problem of target detection in different clutter backgrounds, other practical applications for the problem under consideration can be mentioned. Due to recent advances in technology, development of light-weight small-size millimeter-wave (MMW) radar-based sensors for a myriad of civilian and defense applications has become economically viable [20]. For example, a simple MMW continuous-wave radar system can be designed to be mounted on a vehicle to assess the road condition such as determining the surface roughness, surface wetness, and detecting the existence of an ice or snow layer on the road surface. This information can then be linked to the anti-lock brake or traction control systems of the vehicle. In Section IV, this specific example accompanied by experimental results is described in detail.

Let us consider a nonpolarimetric radar system. This radar is to measure the radar cross section (RCS) or backscattering coefficient of a point or a distributed target chosen from $N_{t}$ different types of targets and then to identify the target. Since the target identification has to be accomplished based on a single measured data point, it is desired that the backscatter power from different targets be as distinct as possible. To accomplish this task, we are free to choose the polarization configurations of the transmit and receive antennas which result in RCS's or backscattering coefficients for different targets that are most distinct. Success of a target discriminator based on the optimal polarization relies on the a priori knowledge of the polarimetric responses of the desired targets. Knowing the scattering matrices of point targets or the covariance matrices of the distributed targets, the RCS's or the backscattering coefficients of the targets for a given pair of transmit and receive polarizations can be determined analytically using

$$
\begin{aligned}
& \sigma_{n}\left(\psi_{r}, \chi_{r}, \psi_{t}, \chi_{t}\right) \\
& \quad=4 \pi \tilde{B}\left(\hat{P}_{r}, \hat{P}_{t}\right) C_{n} B^{*}\left(\hat{P}_{r}, \hat{P}_{t}\right) \quad n \in\left\{1, \cdots, N_{t}\right\}
\end{aligned}
$$

where $\psi_{i}$ and $\chi_{i}$ are the tilt and ellipticity angles of polarization ellipse for transmit $(i=t)$ and receive $(i=r)$ antennas. In (1), $C_{n}$ is the covariance matrix of the $n$th target given by

$$
C_{n}=\left(\begin{array}{cccc}
\left\langle S_{v v} S_{v v}^{*}\right\rangle & \left\langle S_{v v} S_{v h}^{*}\right\rangle & \left\langle S_{v v} S_{h v}^{*}\right\rangle & \left\langle S_{v v} S_{h h}^{*}\right\rangle \\
\left\langle S_{v h} S_{v v}^{*}\right\rangle & \left\langle S_{v h} S_{v h}^{*}\right\rangle & \left\langle S_{v h} S_{h v}^{*}\right\rangle & \left\langle S_{v h} S_{h h}^{*}\right\rangle \\
\left\langle S_{h v} S_{v v}^{*}\right\rangle & \left\langle S_{h v} S_{v h}^{*}\right\rangle & \left\langle S_{h v} S_{h v}^{*}\right\rangle & \left\langle S_{h v} S_{h h}^{*}\right\rangle \\
\left\langle S_{h h} S_{v v}^{*}\right\rangle & \left\langle S_{h h} S_{v h}^{*}\right\rangle & \left\langle S_{h h} S_{h v}^{*}\right\rangle & \left\langle S_{h h} S_{h h}^{*}\right\rangle
\end{array}\right)
$$

and $B\left(\hat{P}_{r}, \hat{P}_{t}\right)$ is the polarization cross-product vector given by

$$
B\left(\hat{P}_{r}, \hat{P}_{t}\right)=\left(\begin{array}{l}
\hat{P}_{v}^{r} \hat{P}_{v}^{t} \\
\hat{P}_{v}^{r} \hat{P}_{h}^{t} \\
\hat{P}_{h}^{r} \hat{P}_{v}^{t} \\
\hat{P}_{h}^{r} \hat{P}_{h}^{t}
\end{array}\right)
$$

In (3), the polarization unit vector $\hat{P}_{i}$ is calculated from

$$
\hat{P}_{i}=\frac{\hat{v}+a_{i} e^{j \delta_{i}} \hat{h}}{\sqrt{1+a_{i}^{2}}}
$$

where $a_{i}$ and $\delta_{i}$ in terms of the ellipticity and tilt angles can be obtained from [21]

$$
\begin{aligned}
\tan \delta_{i} & =\frac{\tan 2 \chi_{i}}{\sin 2 \psi_{i}} \\
a_{i} & =\tan \left[\frac{1}{2} \cos ^{-1}\left(\cos 2 \chi_{i} \cos 2 \psi_{i}\right)\right] .
\end{aligned}
$$




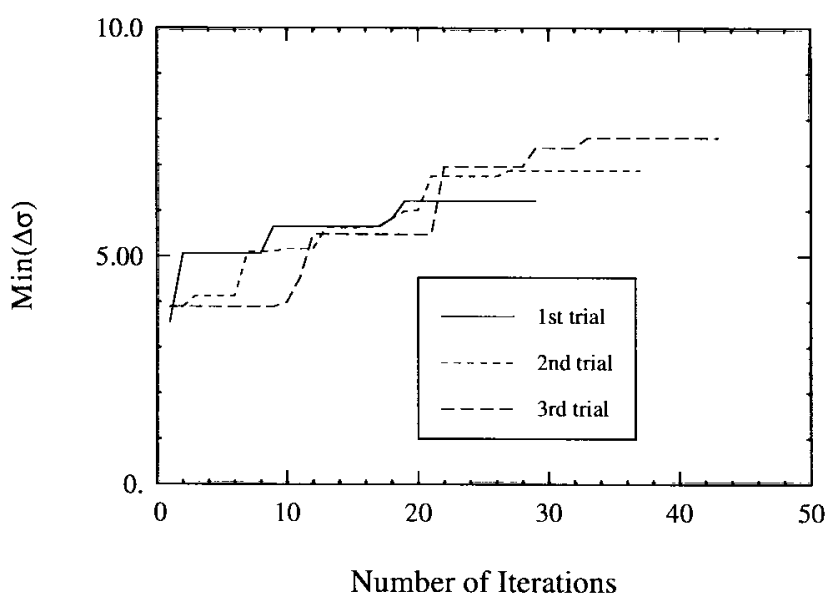

(a)

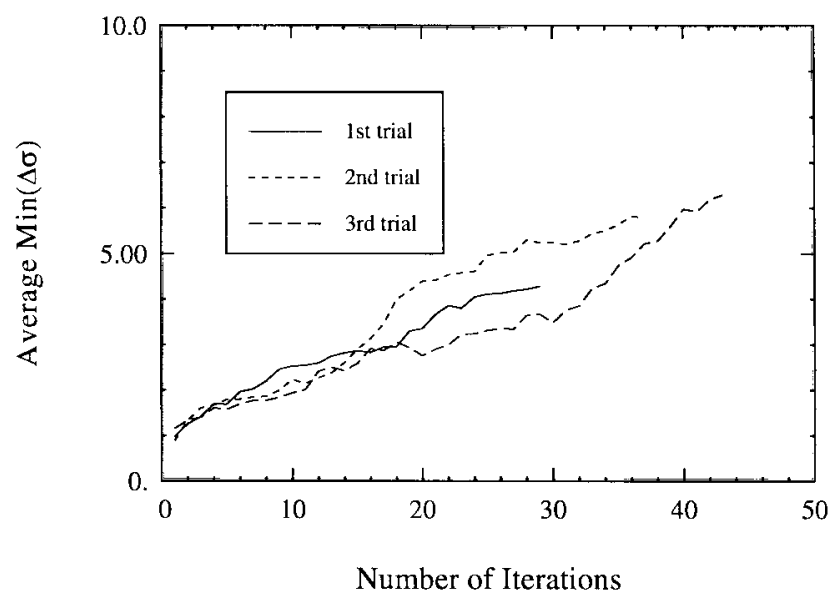

(b)

Fig. 2. The convergence performance of the GA in characterizing the optimum polarization for classification of the four-point targets.

It should be noted that determination of $\delta_{i}$ from (4) is not unique and often leads to confusion. The following constraints remove the ambiguity in determination of $\delta_{i}$

$$
\begin{aligned}
& \text { if } \chi_{i} \leq 0 \Rightarrow \delta_{i} \leq 0 \\
& \text { if } \chi_{i} \geq 0 \Rightarrow \delta_{i} \geq 0 \\
& \text { if } \psi_{i}>0 \Rightarrow\left|\delta_{i}\right|<\pi / 2 \\
& \text { if } \psi_{i}<0 \Rightarrow\left|\delta_{i}\right|>\pi / 2 .
\end{aligned}
$$

The search in the optimization problem has to be performed for pairs of $\left(\psi_{t}, \chi_{t}\right)$ and $\left(\psi_{r}, \chi_{r}\right)$ in a four-dimensional (4-D) vector space bounded by a $-90 \leq \psi_{i} \leq+90,-45 \leq \chi_{i} \leq$ $+45(i \in\{r, t\})$ so that $\sigma_{n}$ 's for $n=1, \cdots, N_{t}$ are most distinct. Before the optimization problem can be formulated, this objective function must be expressed in a mathematical form. In the problem of two-target classification $\left(N_{t}=2\right)$, the objective function is maximization of the ratio

$$
r\left(\psi_{r}, \chi_{r}, \psi_{t}, \chi_{t}\right)=\frac{\sigma_{2}}{\sigma_{1}}
$$

or difference

$$
d_{12}\left(\psi_{r}, \chi_{r}, \psi_{t}, \chi_{t}\right)=\left|f\left(\sigma_{2}\right)-f\left(\sigma_{1}\right)\right|
$$

where $f(\cdot)$ is a user defined function. The problem in this case is well defined and an analytical solution for the constrained maximization problem can be obtained [12]. However, for more than two targets a definition for the objective function is not straightforward and the optimization problem becomes very complex. Noting that the objective function defined by (5) is a subset of (6), we adopt functional (6) as a measure of distance between two functions. The difficulty arises from the fact that a set of input parameters that maximizes the distance between the $m$ th and $n$th targets $\left(d_{m n}\right)$ may minimize the distance between two other targets. In this case, even expressing the objective function mathematically seems rather difficult. Using an analogy between the backscatter functions and points confined within a planar geometrical boundary, the following definition for the objective function is derived. Basically, the optimum point in the 4-D vector space is a point such that the minimum distance among all possible pairs of functions is maximized. Mathematically, the objective function can be expressed as

$$
\begin{gathered}
\operatorname{Maximize}\left\{\operatorname{Min}\left\{d_{m n}\left(\psi_{r}, \chi_{r}: \psi_{t}, \chi_{t}\right)\right\}\right. \\
\left.\forall m, n \in\left\{1, \cdots, N_{t} ; m \neq n\right\}\right\}
\end{gathered}
$$

subject to

$$
\psi_{r}, \psi_{t} \in[-\pi / 2, \pi / 2] \quad \chi_{r}, \chi_{t} \in[-\pi / 4, \pi / 4] .
$$

Noting that for $N_{t}$ targets there are $N_{t}\left(N_{t}-1\right) / 2$ distance functions to be computed, analytical determination of the optimum point in the 4-D vector space is very difficult-if not impossible. In this case, the objective function is piecewise continuous with unknown discontinuity points. The optimization problem is similar to the min-max problem [22] for which the standard approach is to transfer the original problem into a smooth but nonlinearly constrained problem. For example, by introducing a new variable $D$, which is a lower bound on all the distance functions $d_{m n}$ 's, we need to

$$
\begin{aligned}
& \operatorname{Maximize}\{D\} \\
& \qquad D \in(0, \infty) ; \quad \psi_{r}, \psi_{t} \in[-\pi / 2, \pi / 2] \\
& \chi_{r}, \chi_{t} \in[-\pi / 4, \pi / 4] .
\end{aligned}
$$

Subject to $d_{m n}\left(\psi_{r}, \chi_{r} ; \psi_{t}, \chi_{t}\right) \geq D, \forall m, n \in 1, \cdots, N_{t}$. Systematic solutions to this problem exist when the distance functions are linear. Other than the special case of linear functions, hybrid optimization methods based on a priori knowledge of the function's discontinuity points and assumptions on the smoothness of the objective function must be tried. In this paper, instead of approaching the optimization problem from a classical point of view, a discrete numerical approach based on GA's will be used.

Another important issue in the optimization problem is the selection of an appropriate function $f$ used in the distance function given by (6). The function can be chosen to be linear, compressor, or expander. A linear function preserves the significance of distance between two RCS's independent of the absolute values of the RCS's, whereas a compressor or an expander function puts more emphasis on the difference when the absolute values of the RCS's are relatively small 
TABLE I

Uniform Discretization Scheme of Poincare Sphere, Where $k(\chi)$ Is the Number Points on Circles of Constant $\chi$

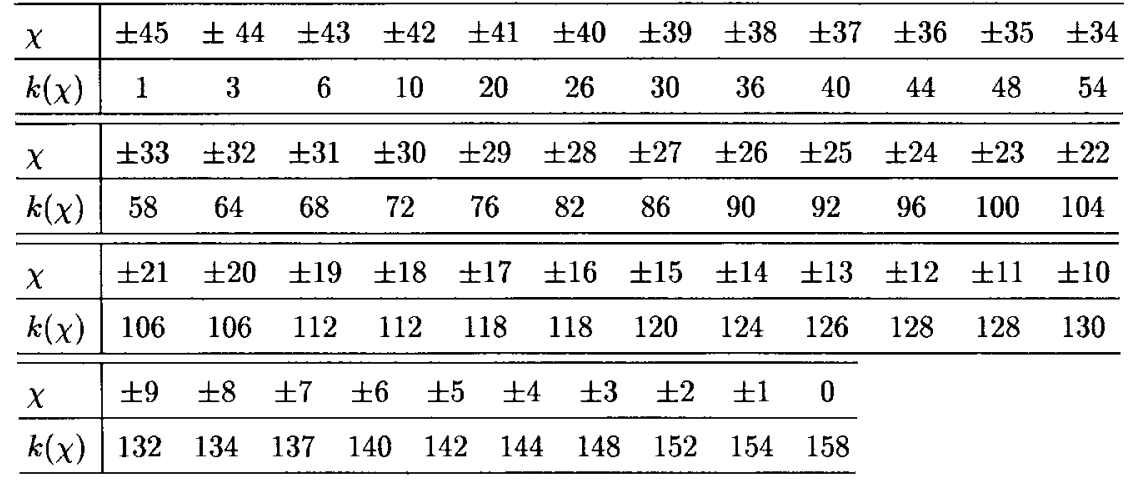

or large, respectively. In dealing with distributed targets, the quantity of interest is the backscattering coefficient $\left(\sigma^{\circ}\right)$ which is proportional to the mean of the backscattered power. The probability density function describing the statistics of backscatter power for most practical cases is exponential. A feature of the exponential distribution is that its mean to standard deviation ratio is unity. Therefore, the uncertainty in estimation of $\sigma^{\circ}$ of a target increases for targets with large $\sigma^{\circ}$ 's. This property requires that the differences between backscattering coefficients of targets with large $\sigma^{\circ}$ 's be larger than those of targets with small $\sigma^{\circ}$ 's. In other words, a compressor function must be used. A logarithmic function is an excellent choice for this application [23]. This can be demonstrated by considering estimation of $\sigma^{\circ}$ using $N$ independent samples. In this case, the standard deviation of the estimate of the mean is $\sigma^{\circ} / \sqrt{N}$. A confidence region for the estimate defined by peak-to-peak variation around the mean for a linear function is $2 \sigma^{\circ} / \sqrt{(N)}$, which increases with increasing $\sigma^{\circ}$. However, for logarithmic functions, the same confidence region will be mapped to

$$
\begin{aligned}
C R & =10 \log \left(\sigma^{\circ}+\sigma^{\circ} / \sqrt{N}\right)-10 \log \left(\sigma^{\circ}-\sigma^{\circ} / \sqrt{N}\right) \\
& =10 \log \left(\frac{\sqrt{N}+1}{\sqrt{N}-1}\right)
\end{aligned}
$$

which is a constant independent of $\sigma^{\circ}$. Therefore, by maximizing the minimum distance between the backscattering coefficients in decibel scale, the separation between the confidence boundaries of the targets is maximized as well.

\section{OptimizATION PROCEDURE Using A GENETIC ALGORITHM}

In this section, a brief overview of GA's is provided. The interested reader is referred to an excellent tutorial article by Haupt [24]. GA's are probabilistic optimization methods that use an iterative search technique based on ideas from evolutionary principles. The algorithm is based on a number of ad hoc steps including: 1) discretization of the parameter space; 2) development of an arbitrary encoding algorithm to establish a one-to-one relationship between each code and the discrete points of the parameter space; 3) random generation of a trial set known as initial population; 4) selection of high- performance parameters according to the objective function known as natural selection; 5) mating and mutation; and 6) recursion of 4) and 5) until a convergence is reached. Consider an $N$-dimensional optimization problem where $N$ parameters are to be chosen so that the objective function $F$ is extremized. In implementation of GA's each parameter is represented by a gene that is simply the binary representation of the parameter. A trial solution comprised of $N$ genes is referred to as a chromosome. The objective is to find the most-fit chromosome according to the objective function.

The first step in implementing a GA is to discretize the parameter space so that the quantization error in calculation of the objective function is below a tolerable threshold. Oversampling of the parameter space would result in an inefficient optimization procedure. The binary transformation of the discretized parameters can be arbitrary; however, it must be one-to-one and onto. This ensures that for every binary string of say $n_{p}$ digits, there is a parameter $p$ in the discrete space.

The optimization procedure starts with initialization of the population. The population size is provided by the user and a population of the given size is generated randomly. A simplified flow chart of a GA is shown in Fig. 1. Once the initial population is created, their performance (fitness) is calculated according to the objective function. Chromosomes then go through a selection operation where only the most fit chromosomes are preserved. The selection operator can either be stochastic or deterministic. In the stochastic approach known as "roulette wheel selection method" [25], a portion of the original chromosomes survive with a probability density function directly proportional to the objective function values associated with the chromosomes. In the deterministic approach, the chromosomes are ranked according to their objective function values and the top 50\% or high ranked (above certain threshold) chromosomes are kept [24]. In this paper, we adopted the deterministic selection approach.

The next step in the optimization procedure is the mating of the survived chromosomes, which will be referred to as parents. This step is accomplished using an operator known as crossover operator. The crossover operator acts on parent chromosomes and generates offsprings by swapping portions of the genetic codes of the parents. In this algorithm, a single crossover point is selected randomly and two offsprings are 
TABLE II

The Optimum Polarization States Derived by the GA Using Three Independent Initial Population Sets for Discriminating Four-Point Targets and Their Radar Cross Sections at the Optimum Polarizations

\begin{tabular}{c|ccccccccc}
\hline & $\psi_{r}$ & $\chi_{r}$ & $\psi_{t}$ & $\chi_{t}$ & $\sigma_{s}$ & $\sigma_{c}$ & $\sigma_{d}$ & $\sigma_{v}$ & $\operatorname{Min}(\Delta \sigma)$ \\
\hline 1st & 47.1 & -10.0 & -44.33 & -8.0 & -16.55 & -1.59 & 10.9 & 4.76 & 6.2 \\
2nd & -46.3 & 9.0 & 54.0 & 6.0 & -3.84 & -10.73 & 10.90 & 3.21 & 6.9 \\
3rd & -47.5 & 4.0 & 52.1 & 2.0 & -4.43 & -14.30 & 10.96 & 3.36 & 7.6 \\
\hline
\end{tabular}

TABLE III

The Optimum Polarization States Derived by the Ga Using Three Independent Initial Population Sets for Discriminating Four Distributed Targets and Their Radar Cross Sections at the Optimum Polarizations

\begin{tabular}{c|ccccccccc}
\hline & $\psi_{r}$ & $\chi_{r}$ & $\psi_{t}$ & $\chi_{t}$ & $\sigma_{d}^{\circ}$ & $\sigma_{w}^{\circ}$ & $\sigma_{i}^{\circ}$ & $\sigma_{s}^{\circ}$ & $\operatorname{Min}(\Delta \sigma)$ \\
\hline 1st & -87.3 & 9.0 & -5.6 & 32.0 & -21.2 & -33.45 & -27.25 & -15.25 & 5.98 \\
2nd & 16.8 & 17.0 & -86.2 & 4.0 & -22.10 & -34.43 & -28.20 & -15.92 & 6.10 \\
3rd & -86.2 & 5.0 & 15.6 & 22.0 & -21.88 & -34.25 & -27.94 & -15.64 & 6.06 \\
\hline
\end{tabular}

generated. The binary codes of the children to the left of the crossover point are the same as those of their parents and their remaining codes are obtained by swapping the binary codes of the parents. The purpose of mating is to construct new chromosomes with higher fitness values. The last step before fitness evaluation of the new chromosomes is mutation. The mutation process is carried out on a chromosome by changing a bit from zero to one or vice versa. The purpose of mutation process is to keep population diversity among the new generations, which, in effect, provides new search regions for the optimization algorithm. Probability of bit mutation per iteration should be kept low enough so that the highly fit chromosomes are not destroyed. Bit mutation with probability of less than $1 \%$ is recommended. After mutation performance of the new chromosomes are evaluated and the process is repeated until a convergence is reached.

An encoding procedure must be established to provide a one-to-one mapping between the discretized parameter space and a binary code. First, let us consider the discretization of the parameter space. One important issue in a discretization process is the resolution. A fine resolution increases the accuracy at the expense of increasing the search domain. It is noted that the radar backscatter cross sections for point targets and backscattering coefficients for distributed targets are very smooth functions of the transmitter and receiver polarization angles $(\psi, \chi)$. Thus, an angular resolution on the order of $1^{\circ}$ is sufficient for most practical applications. Another issue is the discretization scheme. Noting that for almost circular polarizations the sensitivity of the RCS or the backscattering coefficient to variations in the tilt angle is very low, a uniform discretization of the parameter space seems illogical. There is a one-to-one correspondence between the polarization state of the receiver or transmitter and a point on the surface of Poincare sphere, which suggests a uniform discretization of the sphere surface. The area of a pixel on the surface of a Poincare sphere is given by

$$
\Delta A=4 r^{2} \cos 2 \chi \Delta \chi \Delta \psi
$$

Requiring equal pixel area independent of $\psi$ and $\chi$, the total number of pixels are obtained from

$$
N_{p}=\frac{\pi}{\cos 2 \chi \Delta \chi \Delta \psi} .
$$

To establish a one-to-one correspondence between the discrete points on the surface of a Poincare sphere and a complete set of binary codes, $N_{p}$ must be equal to $2^{n}$ for some integer $n$. Choosing a fixed resolution for $\Delta \chi=1^{\circ}$, the resolution for tilt angle as a function of $\chi$ can be obtained from

$$
\Delta \psi=\frac{10314 \times 2^{-n}}{\cos 2 \chi} \text { (deg.) }
$$

In order to achieve almost equally spaced points on the sphere surface, $n=13$ can be chosen. Guided by (9) and after some inspections the number of discrete points $k(\chi)$ on circles of constant $\chi$ is obtained and reported in Table I. Note that according to this discretization scheme

$$
\sum_{m=-45}^{45} k(m)=8192
$$

as expected. Simply by numbering the points from zero (south pole) to 8191 (north pole), each polarization can be assigned to a decimal number. The relationship between the polarization state $(\psi, \chi)$ and its decimal representation $p$ is given by

$$
\text { For } \begin{aligned}
m & =-45 \text { to } 45 \\
\chi & =m \\
\psi & =\frac{180 \ell}{k(m)}, \quad \ell=0, \cdots, k(m)-1 . \\
p & =\sum_{m^{\prime}=-45}^{m-1} k\left(m^{\prime}\right)+\ell
\end{aligned}
$$

Now, expressing $p$ in terms of a 13-bit binary code, we have established a one-to-one correspondence between a polarization state and a binary code. Combining the binary codes for the transmit and receive polarization states, a 26-bit binary 
code is obtained, which represents a discrete point within the domain of the input parameter space. According to our discretization scheme there are a total of 67108864 possible chromosomes in the discretized parameter space.

\section{RESULTS AND DISCUSSIONS}

In this section, performance of the GA in characterization of optimum radar polarizations is studied by considering two examples. In the first example, the optimization problem for four-point targets based on synthetic data is examined. The second example is concerned with a practical application of millimeter-wave radar sensors for characterization of road surface conditions. The GA is used to determine the polarization states of the transmitter and receiver of a nonpolarimetric millimeter-wave radar for the purpose of classifying the road surface into four categories: 1) dry asphalt; 2) wet asphalt; 3 ) ice-covered asphalt; and 4) snow-covered asphalt. For the point target classification example, we chose four targets with similar RCS's. These include a metallic sphere, a composite target made up of two thin cylinders $\lambda / 8$ apart oriented $45^{\circ}$ with respect to the reference vertical direction, a dihedral corner reflector, and a thin vertical cylinder whose scattering matrices are, respectively, given by

$$
\begin{aligned}
S_{s} & =\left(\begin{array}{ll}
1 & 0 \\
0 & 1
\end{array}\right), \quad S_{c}=\frac{\sqrt{2}}{2}\left(\begin{array}{cc}
1 & -j \\
-j & 1
\end{array}\right) \\
S_{d} & =\left(\begin{array}{cc}
1 & 0 \\
0 & -1
\end{array}\right), \quad S_{v}=\left(\begin{array}{ll}
1 & 0 \\
0 & 0
\end{array}\right) .
\end{aligned}
$$

The GA developed in this paper allows the user to choose the number of population and to initiate the algorithm with different sets of initial population. The population number must be chosen according to the size of discretized parameter space (in this case $2^{26}$ ). If the population number is chosen too small, the algorithm may converge to a weak local maxima. On the other hand, the algorithm becomes numerically very inefficient if a large population number is chosen. For this problem it was found that a population number on the order of 70-100 provides satisfactory results. Fig. 2(a) shows the convergence rate of the GA for the four-point target problem with three statistically independent initial population sets. In this figure, the performance measure, which is simply the minimum RCS difference (in decibels) between any two targets among the aforementioned four targets, is displayed. The solutions $\left(\psi_{r}, \chi_{r}, \psi_{t}, \chi_{t}\right)$ for the three different initial population sets together with the RCS's of the targets and the maximized minimum RCS difference $(\operatorname{Min}(\Delta \sigma))$ at the optimum polarizations are reported in Table II. Although at the third trial a better solution is obtained it can be seen that in all three cases the algorithm finds comparable and reasonable results. The results clearly indicate that there exist polarizations for optimal multiple target classification even for targets with comparable RCS's. To examine whether the algorithm is converging to a maximum or it is randomly searching the parameter space, the average of the performance measure of all chromosomes for each generation as the process evolves can be studied. Fig. 2(b) shows an increasing trend

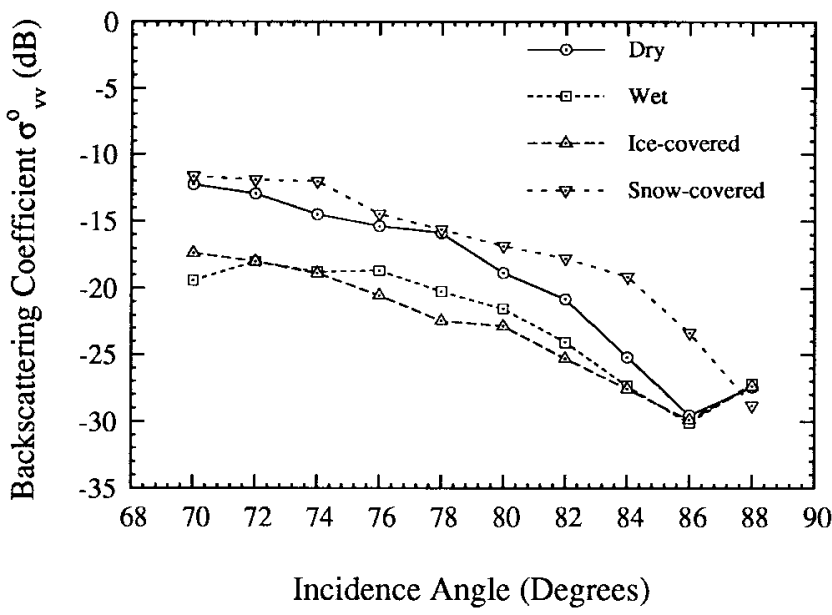

(a)

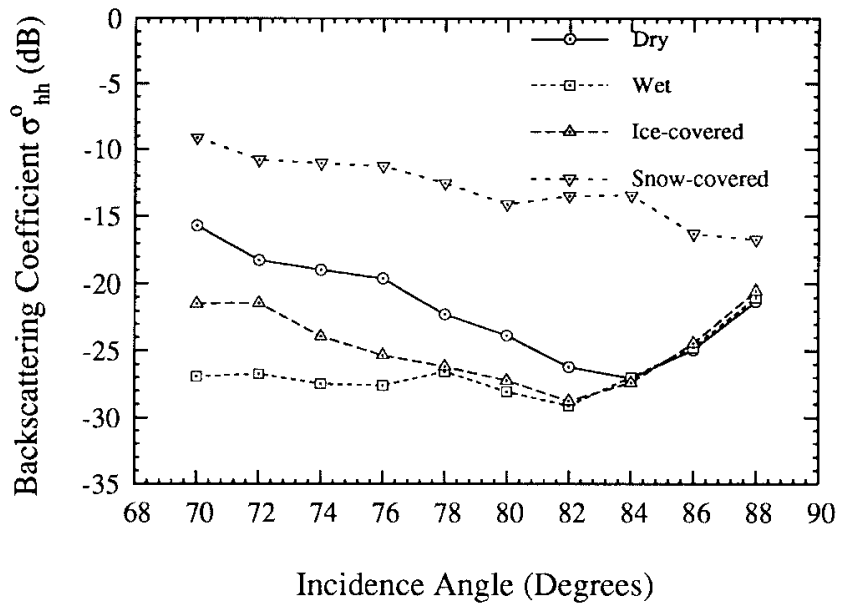

(b)

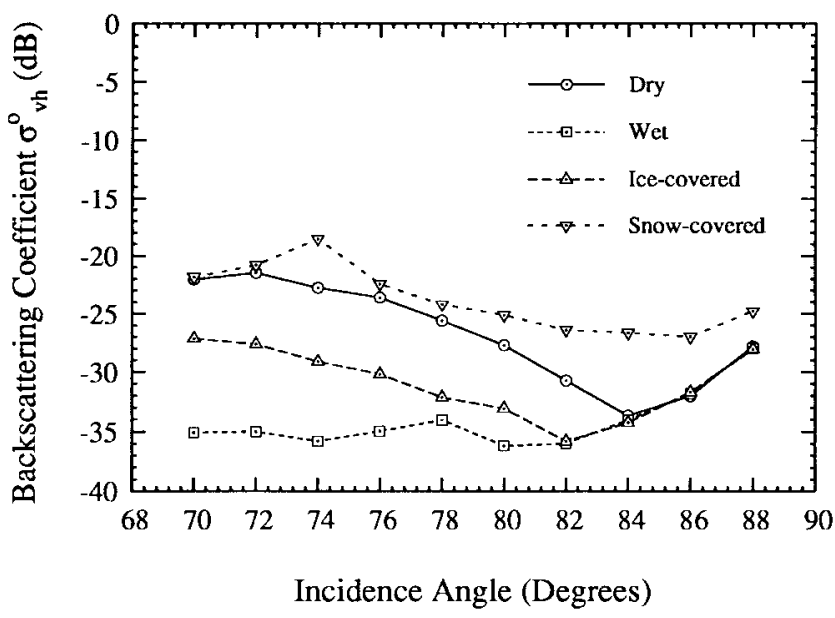

(c)

Fig. 3. The measured backscattering coefficients. (a) $\sigma_{v v}$, (b) $\sigma_{h h}$, and (c) $\sigma_{v h}$ of dry asphalt, wet asphalt, ice-covered asphalt, and snow-covered asphalt at $94 \mathrm{GHz}$, as a function of incidence angle.

for the average of the performance measure as the algorithm converges.

Next we considered a radar classification problem for four distributed targets. In this example a practical application is in mind. Basically, we are seeking the optimal design of 


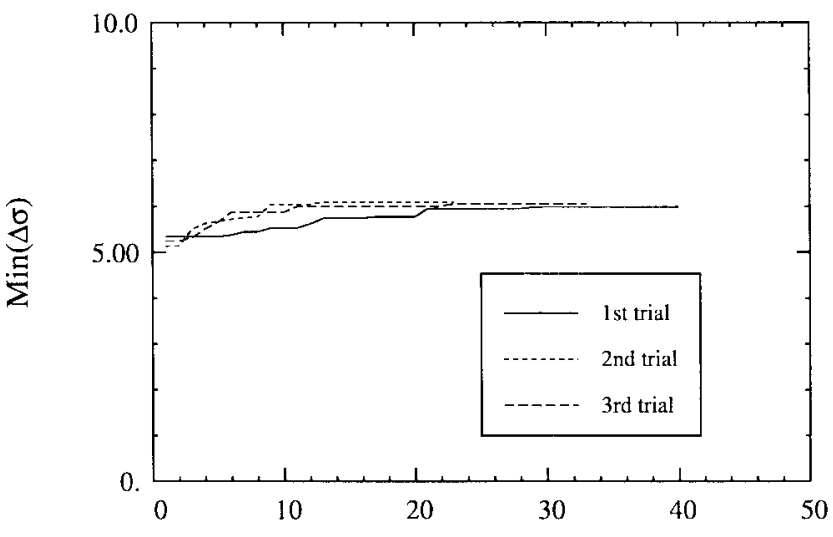

Number of Iterations

(a)

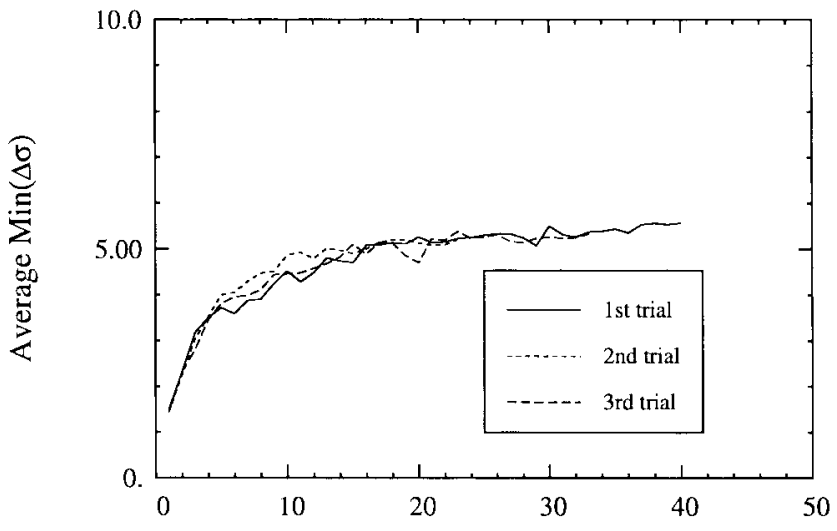

Number of Iterations

(b)

Fig. 4. The convergence performance of the GA in characterizing the optimum polarization for classification of the four distributed targets.

an affordable millimeter-wave radar sensor for automotive applications that can assess traction of road surfaces. For this purpose, a relatively smooth asphalt surface with rms height of about $0.34 \mathrm{~mm}$ was chosen. The same asphalt surface for four different conditions were considered. These include: 1) dry asphalt; 2) a wet surface condition with water content $0.8 \mathrm{~kg} / \mathrm{m}^{2}$; 3) the asphalt surface covered by an ice layer with thickness $1.4 \mathrm{~mm}$; and 4) the asphalt surface covered by a snow layer with thickness $6.4 \mathrm{~mm}$ and density 68 $\mathrm{kg} / \mathrm{m}^{3}$. The University of Michigan's fully polarimetric 94$\mathrm{GHz}$ scatterometer was used to characterize the polarimetric responses of the asphalt surfaces. This system operates at 93.5 $\mathrm{GHz}$ with a bandwidth of $0.5 \mathrm{GHz}$. The backscatter measurements were conducted over the incidence angular range $70^{\circ}-88^{\circ}$. To characterize the statistics of the backscatter responses, more than 80 independent spatial samples for each target were collected. Systematic measurement errors such as channel imbalances, antenna cross talks, polarization switches distortions, and radiometric calibration constant are accounted for by using an external calibration procedure [26]. The backscattering coefficients of the four asphalt surfaces as a function of incidence angle are shown in Fig. 3(a) $\left(\sigma_{v v}^{\circ}\right)$, (b) $\left(\sigma_{h h}^{\circ}\right)$, and (c) $\left(\sigma_{v h}^{\circ}\right)$. After the calibration procedure, the covariance matrices of the asphalt surfaces at $74^{\circ}$ were fed to the GA and the results are reported in Table III for three different initial population sets. For each case, the approximate computer time to arrive at an optimal solution using this algorithm (GA) is less than 1 min on a SPARC 20 workstation. In this table, the subscripts $d, w, i$, and $s$ denote dry, wet, ice, and snow, respectively. Similar performances are obtained in all three cases, although three different solutions are obtained. A minimum $\sigma^{\circ}$ difference of $6 \mathrm{~dB}$ among different surface road conditions is obtained that allows for a reliable target classification. Fig. 4(a) and (b) shows the convergence behavior of the algorithm in this case. As expected, the average of performance measure for each generation improves as the algorithm approaches the convergence point.

\section{CONCLUSIONS}

Determination of polarization states of the transmitter and receiver antennas of a nonpolarimetric radar for optimal target classification was considered in this paper. Assuming that the polarimetric responses of the targets under consideration are known a priori, the optimization problem was formulated to search for a pair of polarization configurations so that at these polarizations the minimum separation of the backscatter power between any two targets among all possible targets is maximized. To achieve this goal a global search routine based on a GA was used. The success of the GA was demonstrated by applying the algorithm to two cases of point and distributed targets. The issues regarding the user defined parameters of the algorithm such as the population size and probability of mutation as well as the convergence property of the algorithm were discussed. Optimal polarization synthesis for a nonpolarimetric millimeter-wave radar system considered for assessing traction of road surfaces was carried on based on a set of polarimetric backscatter measurements of asphalt surfaces under different physical conditions at $94 \mathrm{GHz}$.

\section{REFERENCES}

[1] E. M. Kennaugh, "Effect of type of polarization on echo characteristics," Antenna Lab., The Ohio State Univ., Rep. 389-5, Sept. 1950.

[2] J. R. Huynen, "Phenomenological theory of radar targets," Ph.D. dissertation, DrukkerijBonder-Offset, N.V., Rotterdam, The Netherlands, 1970.

[3] A. Hendry and G. C. McCormick, "Deterioration of circular polarization clutter cancellation anisotropic precipitation media," Electron. Lett., vol. 10, pp. 165-166, 1974.

[4] G. C. McCormick and A. Hendry, "Polarization related parameters for rain: Measurements obtained by radar," Radio Sci., vol. 11, pp. 731-740, Aug./Sept. 1976.

[5] W. M. Boerner, M. B. El-Arini, C. Y. Chan, and P. M. Mastoris, "Polarization dependence in electromagnetic inverse problems," IEEE Trans. Antennas Propagat., vol. 22, pp. 262-271, Feb. 1981.

[6] J. J. van Zyl, "On the importance of polarization in radar scattering problems," Ph.D. dissertation, California Inst. Technol., Pasadena, CA, 1986.

[7] F. T. Ulaby and C. Elachi, Eds., Radar Polarimetry for Geoscience Applications. Dedham, MA: Artech House, 1990.

[8] I. R. Joughin, D. P. Winebrenner, and D. B. Percival, "Probability density functions for multi-look polarimetric signatures," IEEE Trans. Geosci. Remote Sensing., vol. 32, pp. 562-574, May 1994.

[9] K. Sarabandi, "Derivation of phase statistics of distributed targets from the Mueller matrix," Radio Sci., vol. 27, no. 5, pp. 553-560, 1992.

[10] D. Eliyahu, "Vector statistics of correlated Gaussian fields," Phys. Rev., vol. 74, no. 4, pp. 2881-2892, Apr. 1993. 
[11] M. C. Burl, W. W. Irving, and L. M. Novak, "Optimal polarimetric processing for enhanced target detection," IEEE Trans. Aerosp. Electron. Syst. vol. 29, pp. 234-244, Jan. 1993.

[12] G. A. Ioannidis and D. E. Hammers, "Optimum antenna polarization for target discrimination in clutter," IEEE Trans. Antennas Propagat., vol. 27, pp. 357-363, May 1979.

[13] J. J. van Zyl, N. Engheta, C. H. Papas, C. Elachi, and H. Zebker, "Modeling of backscatter from vegetation layers," in IEEE Geosci. Remote Sensing Symp. Dig., Amherst, MA, Oct. 1985, vol. 1, pp. 389-394.

[14] S. Kirkpatrick, J. C. D. Gelatt, and M. P. Vecchi, "Optimization by simulated annealing," Science, vol. 220, pp. 671-680, 1983.

[15] J. H. Holland, "Genetic algorithms," Sci. Amer., pp. 66-72, July 1992.

[16] K. A. DeJong, "An analysis of the behavior of a class of genetic adaptive systems," Ph.D. dissertation, Univ. Michigan, Ann Arbor, MI, 1975.

[17] E. Michielssen, J. Sajer, S. Ranjithan, and R. Mittra, "Design of lightweight, broad-band microwave absorbers using genetic algorithms,' IEEE Trans. Microwave Theory Tech., vol. 41, no. 6/7, pp. 1024-1031, June/July 1993.

[18] R. L. Haupt, "Thinned arrays using genetic algorithms," IEEE Trans. Antennas Propagat., vol. 42, pp. 993-999, July 1994.

[19] A. Boag, A. Boag, E. Michielssen, and R. Mittra, "Design of electrically loaded wire antennas using genetic algorithms," IEEE Trans. Antennas Propagat., vol. 44, pp. 687-695, May 1996.

[20] Y. Takimoto and M. Kotaki, "Recent development of MM-wave applications in Japan," Microwave J., pp. 214-226, May 1996.
[21] J. D. Kraus, Electromagnetics, 4th ed. New York: McGraw-Hill, 1992, pp. 605.

[22] P. E. Gill, W. Murray, and M. H. Wright, Practical Optimization. London: Academic, 1981, pp. 96-98.

[23] F. T. Ulaby, personal communication.

[24] R. L. Haupt, "An introduction to genetic algorithms for electromagnetics," IEEE Antennas Propagat. Mag., vol. 37, pp. 7-15, Apr. 1995.

[25] D. E. Goldberg, Genetic Algorithms in Search, Optimization, and Machine Learning. Reading, MA: Addison-Wesley, 1989.

[26] A. Nashashibi, K. Sarabandi, and F. T. Ulaby, "A calibration technique for polarimetric coherent-on-receive radar system," IEEE Trans. Antennas Propagat., vol. 43, pp. 396-404, Apr. 1995.

Kamal Sarabandi (S'87-M'90-SM'93), for photograph and biography, see p. 867 of the May 1997 issue of this TRANSACTIONS.

Eric S. Li, for photograph and biography, see p. 1688 of the November 1997 issue of this TransaCTIONS. 